Quantifying the effects of temperature and noise on attention-level using EDA and EEG sensors

\author{
Xue, Z., Yang, L., Rattadilok, P., Li, S., Gao, L.
}

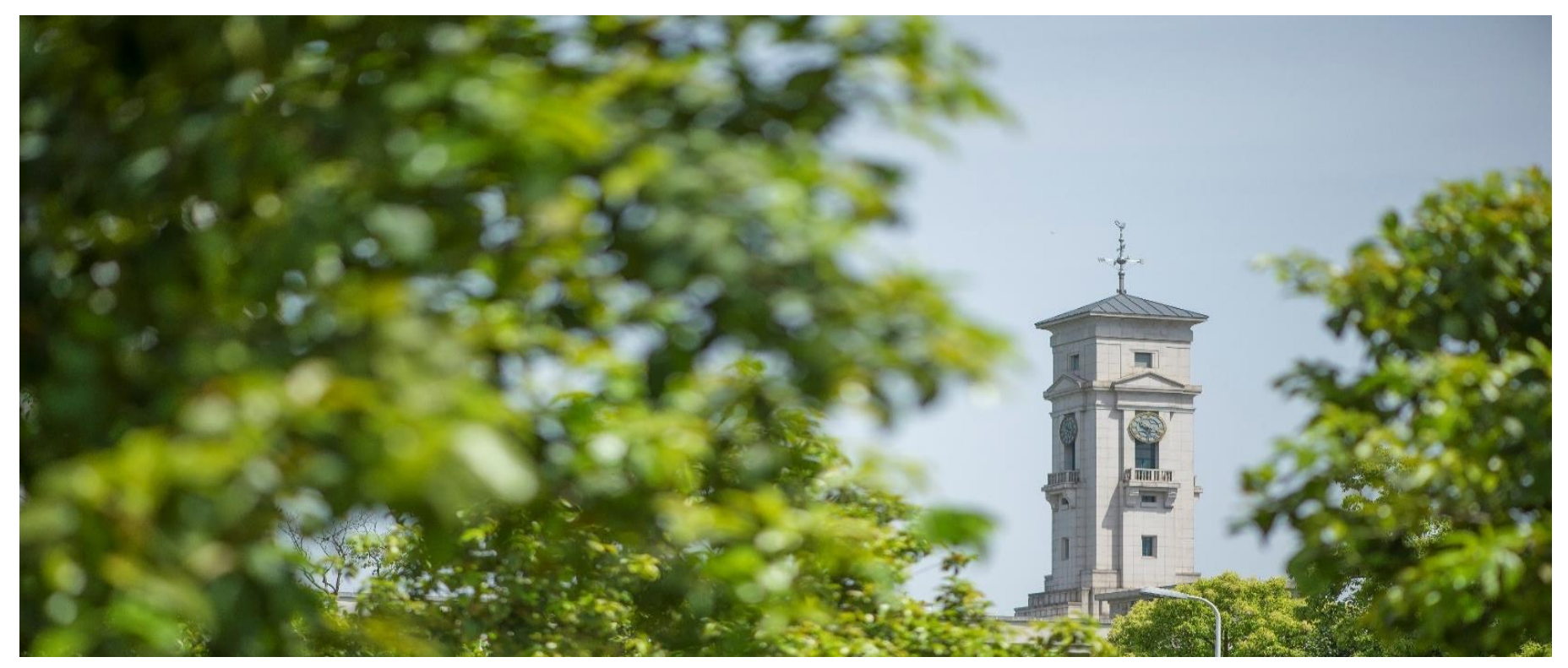


University of Nottingham Ningbo China, 199 Taikang East Road, Ningbo, 315100, Zhejiang, China.

First published 2019

This work is made available under the terms of the Creative Commons Attribution 4.0 International License:

http://creativecommons.org/licenses/by/4.0

The work is licenced to the University of Nottingham Ningbo China under the Global University Publication Licence:

https://www.nottingham.edu.cn/en/library/documents/researchsupport/global-university-publications-licence.pdf 


\title{
Quantifying the Effects of Temperature and Noise on Attention-Level using EDA and EEG Sensors
}

\author{
Zhengrui Xue ${ }^{1}$, Luning Yang ${ }^{2}$, Prapa Rattadilok ${ }^{3}$, Shanshan $\mathrm{Li}^{4}$ and Longyue $\mathrm{Gao}^{5}$ \\ ${ }^{1}$ University of Nottingham Ningbo China, School of Computer Science \\ scyzx1@nottingham.edu.cn \\ ${ }^{2}$ University of Nottingham Ningbo China, School of Mathematical Science \\ smyly1@ nottingham.edu.cn \\ ${ }^{3}$ University of Nottingham Ningbo China, School of Computer Science \\ prapa.rattadilok@nottingham.edu.cn \\ ${ }^{4}$ University of Nottingham Ningbo China, School of Computer Science \\ zy18712@ nottingham.edu. cn \\ ${ }^{5}$ University of Nottingham Ningbo China, School of Computer Science \\ zy18699@nottingham.edu.cn
}

\begin{abstract}
Most people with Autism Spectrum Disorder (ASD) experience atypical sensory modality and need help to self-regulate their sensory responses. Results of a pilot study are presented here where temperature, noise types and noise levels are used as independent variables. Attention-based tests (ABTs), Electrodermal Activity (EDA) and Electroencephalography (EEG) sensors are used as dependent variables to quantify the effects of temperature and noise. Based on the outcome of the analyses, it is feasible to use off-the-shelf sensors to recognize physiological changes, indicating a possibility to develop sensory management recommendation interventions to support people with ASD.
\end{abstract}

Keywords: Autism Spectrum Disorder, Sensory Management, Sensors, Electrodermal Activity, Electroencephalography

\section{INTRODUCTION}

Autism Spectrum Disorder (ASD) is a neurodevelopment disorder characterized by a range of persistent difficulties in social communication, cognitive and repetitive behavior patterns, as well as differences in perceptual processing [1]. Human senses work by acquiring conscious information that allows them to make classifications in their environment [2]. In the United States, one out of 68 children are diagnosed with ASD [3]. Although there is evidence that sensory deficits can impede the development of the ability to socially interact at an early age [4], the sensory training for children with ASD remains unexplored.

Researchers have categorized the heterogeneity of ASD based on behavioral and physical phenotypes [5]. The Diagnostic and Statistical Manual of Mental Disorders, 4th edition (DSM-IV) applies a multi-categorical system in diagnosing ASD. However, 
many studies have indicated that the DSM-IV criteria have limited reliability, poor classification of core symptoms and low predicate ability [6, 7]. Instead of a multi-categorical system, DSM 5th edition (DSM-5) uses a single diagnostic dimension which removes the clinical subtypes in DSM-IV, and determines that symptoms of ASD are best represented in a two-domain model of social-communication deficits and restrictedrepetitive interests/behaviors (RRB) [8]. According to Huerta et al. [9], the specificity of using the new ASD diagnostic criteria is significantly improved. By having any abnormal sensory response to environment or unusual sensory interests within one dimension, researchers can explore the clinical outcomes and treatment response of people with ASD subgroups more efficiently [10].

Some people with ASD have hypersensitive sensory condition and the external stimulus can cause a panic attack or overwhelming sensation [11]. Others could be hyposensitive, with the low perception of temperature and pain. Sensory management plays a critical role in addressing the challenges faced by people with ASD. Sensory technologies are designed to help overcome these restrictions and assist people with ASD to better understand and participate in the social environment [12]. Although the technologies have been shown to support people with ASD effectively [13], no known technological solutions focus on recommending sensory management strategies for people with ASD in real-time. This paper presents a feasibility study of developing a novel sensory management recommendation system, which aims to combine several sensors, working together and providing more comprehensive information.

\section{RELATED WORK}

\subsection{Sound Level Monitors}

People with ASD have motor, sound, sensory and visual impairments, but these impairments are not considered in ASD diagnosis $[14,15]$. Specifically, children with ASD have delayed neural timing, reduced neural stability, and low sensitivity of pitch changes, when compared with their neuro-typical developing peers, and this may negatively influence their communication capability [16].

A sound level meter is a hand-held device equipped with a display and a microphone on the top, e.g. Casella [17]. It can respond to changes in air pressure caused by sound waves, i.e. automatically convert physical vibration into an electrical signal, and thus measure the sound level that travels through the air [17].

Smartphones have recently become an alternative device for measuring sound level. Although limitations exist, some studies suggest that smartphones can replace the traditional sound level assessment devices in the near future [18]. One study suggests that a mobile application that tracks noise level has a significant difference between measured and true noise levels, indicating the low performance for ambient noise measurement in hardware [19]. The same study also shows that the age of a smartphone will also determine its ability to accurately measure noise, however the relation between accuracy and low volatility requires further research.

NIOSH [20] combines the features of high-accuracy sound level meters and noise dosimeters into a mobile application. The application will record the instantaneous 
noise level in the laboratory and calculate an average noise level. LENA or Language Environment Analysis has been used in many ASD researches to collect, manage and analyze the audio record. LENA comes with custom-designed clothing with a pocket to insert LENA recorder. LENA provides counts and percentile data on audio measurements. However, its suitability for clinical applications is so far unproven [21].

\subsection{Physiological Monitors}

\subsubsection{Electrodermal Activity (EDA) Sensor.}

The sweat glands of human are varied and controlled by the sympathetic nervous system. Electrodermal Activity (EDA), also known as Galvanic Skin Response (GSR), refers to changes in sweat gland activity that are reflective of the intensity of our emotional state or arousal. When a person experiences stress or is aroused, moisture collects under the skin, increasing the skin's electrical conductivity which changes the skin temperature and heart rate [22]. Typically, EDA sensors measure the electrical conductance of skin [23] and have been used as an indicator of psychological or physiological arousal.

EDA sensors are widely used for stress assessment and intervention in people with ASD [24, 25]. In addition to an EDA, iCalm [26] also includes a photoplethysmograph (PPG) for the heart rate, 3-axis accelerometer for movements, and an optical infrared thermometer for detecting the skin temperature. One study has tested iCalm for continuous data collection on 7 participants within 48 hours, the results shows that iCalm can run continuously for long periods of time and has similar performance to a clinician standard device [28]. In the electronic device market, Empatica E4 uses the cloud to store user's data from four sensors i.e. 3D accelerometer, PPG sensor, EDA sensor and the skin temperature sensor. Samsung Gear watches lack the EDA sensor but are equipped with Gyro and Barometer sensors, and data from Samsung devices were collected directly by Wi-Fi [27].

It should be noted that EDA cannot determine emotion factors and the EDA sensors need physical contact with the body, which may cause danger during aggressive behaviors. In [28], extensive numbers of wearable solutions for physiological and emotional monitoring for ASD is reviewed.

\subsubsection{Attention Level Sensor.}

Attention is the term that has been defined as the behavioral and cognitive process of selectively concentrating on a discrete aspect of information while ignoring other perceivable information and the allocation of limited cognitive process [29].

The Muse Brain sensor is a lightweight headband that uses electroencephalography (EEG) sensors to monitor human brain activity, which corresponds to attention level [30]. MUSE uses seven precisely calibrated EEG sensors, two on the forehead, two 
behind the ear, and three reference sensors. Signal processing and machine learning techniques are applied to analyze these signals, and visualized as a chart.

The iView X RED eye tracker has been used to detect the attention pattern of people with ASD. In a dark and isolated environment, a small video was provided as a visual stimulus for participants, and an eye tracker was used at a $60 \mathrm{~Hz}$. The subject's gaze pattern is tracked by the device and then analyzed for any atypical patterns in the gaze behavior [31]. However, the device is expensive and requires calibration for every subject. The subject also needs to face the camera, which restricts free movement.

WearCam is a head-mounted eye tracker that measures wide-angle field of view and gaze direction from the perspective of the subject [2]. The video can be analyzed to monitor the subject's focus of attention throughout the session. The mobility of device allows for more natural interactions, but the accuracy is relatively low. Moreover, people who are sensitive to skin contact may not be tolerant the head-mounted devices.

\section{METHODOLOGY}

The aim of the experiment is to measure the attention level of people under different noise levels and temperatures. Attention-level is measured using well-known attentionbased tests (ABTs) including Stroop Color and Word Test (SCWT) [32], Dot Cancellation Test (DCT) [33] and mouse tracking task (MTT) [34]. The speed and the accuracy or the correctness in completing these ABTs are used as the quantitative measurements for attention-level. Within-subjects experimental design [35] is used, i.e. each participant participates in all of the experimental conditions. In minimizing the learning effect, i.e. participants became more familiar with the tasks over time, Latin-square design $[36,37]$ is used to place the order of the tasks for different participants.

The temperature of the experimental room is controlled digitally using an air-conditioner and a heater. A temperature sensor and a sound sensor connected to an Arduino UNO board are used to monitor and record the temperature and the sound level during the experiment. Data from Arduino UNO board is compared with a digital thermostat and the NIOSH app to ensure the accuracy of the measurements. GSR sensor and MUSE headband are used to measure EDA and EEG respectively.

\subsection{Stroop Color and Word Test (SCWT)}

The SCWT [32] is a test widely used to evaluate the ability of the subject to suppress cognitive interference in neuropsychology. In SCWT, participants are provided colored-words with mismatched colors (Fig. 1). Participants must ignore the meaning of the words and identify the color of the word as soon as possible.

\subsection{Dot Cancellation Task (DCT)}

The DCT [33] is used to study visual focused attention and sustained attention. Participants are shown a picture containing a large set of black dots groups with a number of 
black dots including 3 dots, 4 dots and 5 dots on a computer (Fig. 2). They are required to click all the dot groups containing four dots with mouse as quickly as possible.
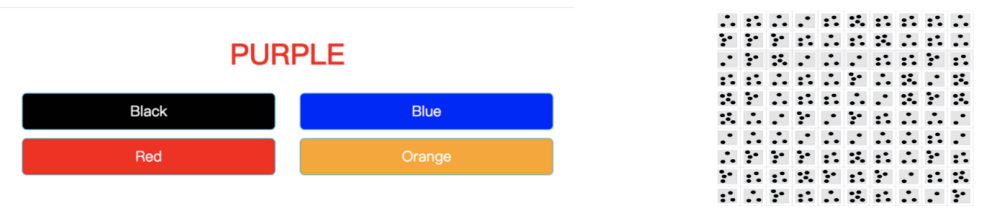

Fig. 1. An example of the SCWT Fig. 2. An example of the DCT

\subsection{Mouse Tracking Task (MTT)}

The MTT was designed to replace eye tracking [34]. Participants are required to follow a curve line by using computer mouse (Fig. 3). The tracking matching rate of the cursor on the curve line is used to evaluate the level of attention.

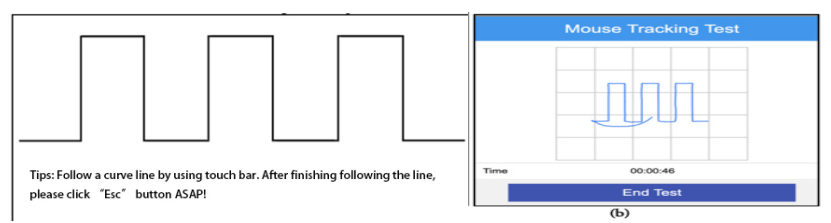

Fig. 3. An example of the MTT and an example of the task result

\section{DISCUSSION}

\subsection{Attention \& Noise Level}

Three different noise types are used i.e. atmospheric noise, man-made noise and industrial noise, to demonstrate the connection between the noise level and human attention level when participants are asked to complete DCT, and MTT. The atmospheric noise is of rainfall. The man-made noise is of hubbub in a coffee shop. The industrial noise is of large industrial fan blowing at high-speed. The participants listen to each of these noise types whilst completing their ABTs under three different loudness i.e. $40 \mathrm{~dB}, 60$ $\mathrm{dB}$ and $80 \mathrm{~dB}$.

\subsubsection{Processing Speed of the Dot cancellation Task (DCT).}

Fig. 4, Fig. 5 and Fig. 6 illustrates processing speed of the DCT when participants are listening to different levels of atmosphere noise, industrial noise and man-made noise respectively. The processing speed is calculated by the formula. 


$$
\mathrm{V}=\frac{\mathrm{N}}{\mathrm{T}}
$$

$\mathrm{V}$ is the processing speed of the DCT i.e. click per second. The higher the V value, the higher the performance of the participant. $\mathrm{N}$ is the number of 4 dot groups the participant processed. $\mathrm{T}$ is the total completion time in seconds. The red dots in the figures represent the averages processing speed under different types of noise and levels of noise, which are also summarized in Table 1 and Fig. 7.

Table 1. Average Processing Speed of the DCT under three different noise levels.

\begin{tabular}{|l|l|l|l|}
\hline & $40 \mathrm{~dB}$ & $60 \mathrm{~dB}$ & $80 \mathrm{~dB}$ \\
\hline Type 1 Atmospheric Noise & 0.3840 & 0.5364 & 0.7324 \\
\hline Type 2 Industrial Noise & 0.8526 & 0.7560 & 0.6421 \\
\hline Type 3 Man-made Noise & 0.7030 & 0.8567 & 0.8126 \\
\hline
\end{tabular}

As shown in Table 1., average processing speed increases with the increasing levels of noise i.e. from $40 \mathrm{~dB}$ to $80 \mathrm{~dB}$ when participants are listening to the atmospheric noise and man-made noise whilst completing the ABTs. The average processing speeds under man-made noise are higher for all of the three levels of noise, than the average processing speed under the atmospheric noise. This indicates that the man-made noise may have a positive influence on the performance of participants in the DCT. However, the average processing speed decreases significantly under the industrial noise.

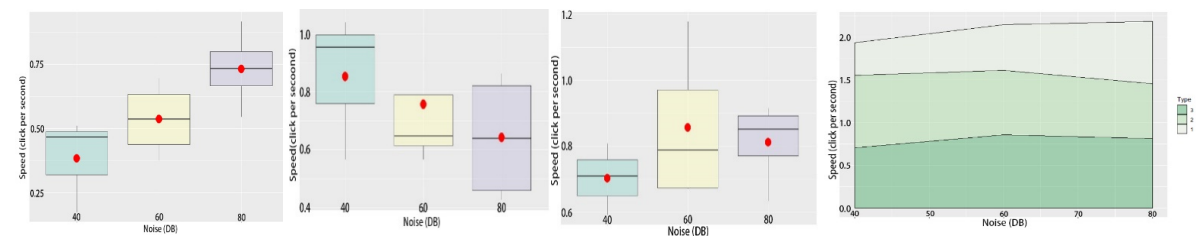

Fig. 4. DCT's processing speed under atmosphere noise. Fig. 5. Under industrial noise.

Fig. 6. Under man-made noise. Fig. 7. Avg. of processing speed.

As shown in Fig. 4, Fig. 5 and Fig. 6, different noise types significantly affect the performance of the DCT. Fig. 7 illustrates the changes in average processing speed when participants are listening to different noise types at different loudness i.e. $40 \mathrm{~dB}, 60 \mathrm{~dB}$, and $80 \mathrm{~dB}$. Noise type 1, 2 and 3 represents atmosphere noise, industrial noise and manmade noise, and the height of each colour represents the mean of processing speed of each noise type under different levels of noise. As illustrated, the processing speed is at its lowest when the atmosphere noise is used at $40 \mathrm{~dB}$, and the processing speed is at its highest when the man-made noise is used at $60 \mathrm{~dB}$.

\subsubsection{Matched Rates of the Mouse Tracking Task (MTT).}

$$
R_{M}=\frac{M}{T}
$$

$R_{M}$ is the matched rate of the testing image. $\mathrm{M}$ is the number of black pixels that match the original image. $\mathrm{T}$ is the total number of black pixels of the test image. 
In the group scenario, 4 participants on 4 computers are sitting in the same room to complete the ABTs. In the individual scenario, the participant is sitting alone in room to the complete the ABTs. Table 2 contains the average match rates under different types of noise and levels of noise.

Table 2. Match Rate - of the group and individual MTT under three different noise levels.

\begin{tabular}{|l|l|l|l|}
\hline & $40 \mathrm{~dB}$ & $60 \mathrm{~dB}$ & $80 \mathrm{~dB}$ \\
\hline Group-Atmosphere Noise & 0.6373 & 0.6583 & 0.7078 \\
\hline Type 1 Atmospheric Noise & 0.6483 & 0.6531 & 0.6380 \\
\hline Type 2 Industrial Noise & 0.6483 & 0.5967 & 0.5940 \\
\hline Type 3 Man-made Noise & 0.6483 & 0.5693 & 0.6437 \\
\hline
\end{tabular}

From Table 2, there is no significant difference in the average match rate between the group scenario and the individual scenario when participants are completing the ABTs whilst listening to atmospheric noise at any loudness. Fig. 8 and Fig. 9 illustrate the box whisker plots showing the performance results of MTT from the group scenario and individual scenario respectively under different atmospheric noise levels.
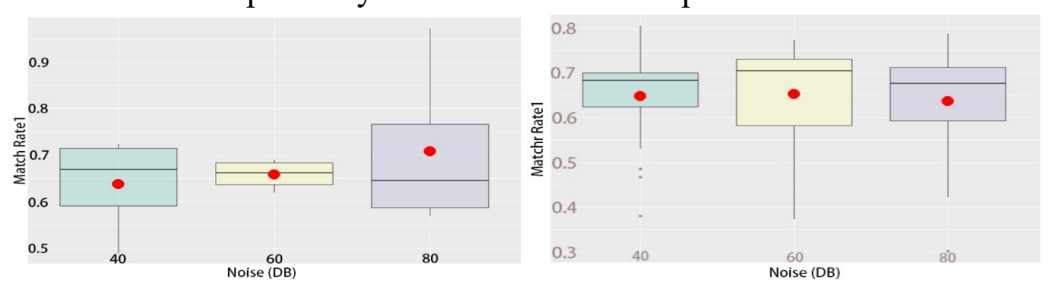

Fig. 8. Results of Matched Rate for group MTT under atmosphere noise. Fig. 9. For individual.

As shown in Fig. 8, there is a slight increase in the match rate of the group scenario when the level of atmospheric noise increases from $40 \mathrm{~dB}$ to $80 \mathrm{~dB}$. However, for the match rate of the individual scenario as shown in Fig. 9, there is only a difference of 0.01 between different levels of noise, which can be deemed negligible. Fig. 10 and Fig. 11 shows the performance results of the MTT for the individual scenario when participants are listening to different noise levels of industrial noise and man-made noise respectively.

As shown in Fig. 10 and Fig. 11, when the individual participants are listening to the industrial noise and man-made noise, the match rates decrease by about 0.2 when the noise level changes from $40 \mathrm{~dB}$ to $60 \mathrm{~dB}$. It can be inferred that the effect of the atmosphere noise on the performance of participants in MTT is barely noticeable, but different noise type can affect the performance of the participants in different ways in MTT.

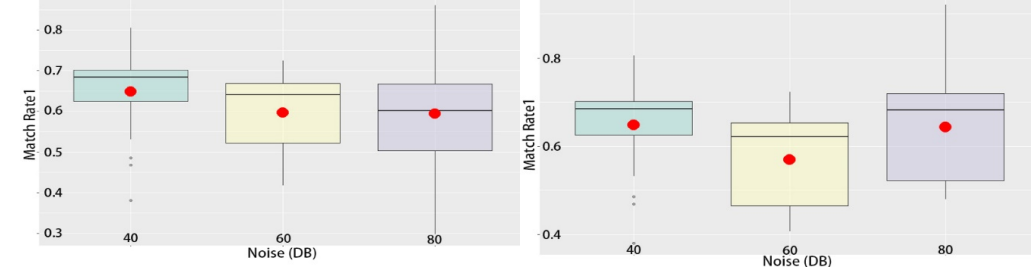

Fig. 10. Matched Rates - individual MTT under industrial noise. Fig. 11. Under man-made noise. 


\subsubsection{Calmness Rates of the EEG Device-Muse.}

Table 3 contains the average calmness rate under different types of noise and levels of noise. Participants were most calm when listening to man-made noise at $60 \mathrm{~dB}$ while completing the ABTs. Participants were least calm when listening to industrial noise at $80 \mathrm{~dB}$ while completing the ABTs. It should be noted that there is no significant difference in the calmness rate when participants are listening to atmospheric noise at different noise levels.

Table 3. Calmness rate of the participants - individual MTT under three different noise levels.

\begin{tabular}{|l|l|l|l|}
\hline & $40 \mathrm{~dB}$ & $60 \mathrm{~dB}$ & $80 \mathrm{~dB}$ \\
\hline Type 1 Atmospheric Noise & 0.5257 & 0.5055 & 0.5047 \\
\hline Type 2 Industrial Noise & 0.5257 & 0.4865 & 0.4004 \\
\hline Type 3 Man-made Noise & 0.5257 & 0.6563 & 0.4313 \\
\hline
\end{tabular}

Fig. 12, Fig. 13 and Fig. 14 show the calmness rates of the participants under different level of atmospheric noise, industrial noise, and man-made noise respectively. The red dots in the figures represent the average calmness rates of the participants under different types of noise and levels of noise.

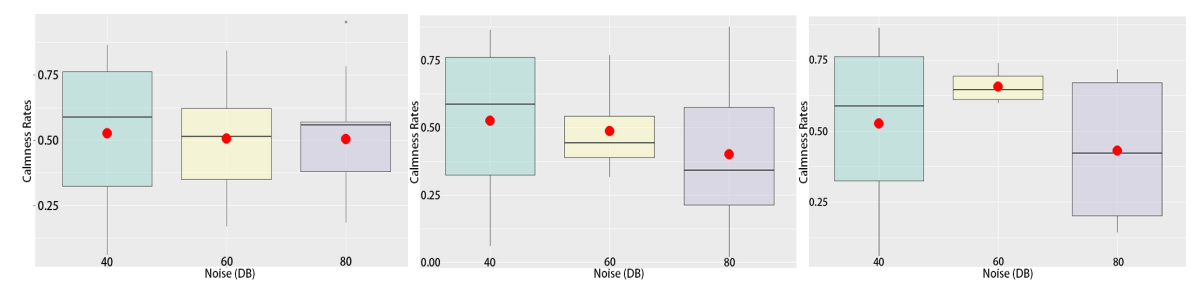

Fig. 12. Calmness rates under atmosphere noise. Fig. 13. Industrial noise. Fig. 14. Man-made noise.

\subsection{Attention \& Temperature Level}

Three different temperatures are used i.e. $17^{\circ} \mathrm{C}, 24^{\circ} \mathrm{C}$ and $31^{\circ} \mathrm{C}$, to demonstrate the connection between the temperatures and human attention level when participants are asked to complete the SCWT and the DCT. The average measured values of the temperatures from the experiment are $17.38,24.13$ and 30.50 respectively.

\subsubsection{Single-Click Times in the Stroop Color and Word Test (SCWT).}

\subsubsection{The limitation of no warm-up question.}

Fig. 15 shows that the amount of time the participants take to answer each question varies greatly, particularly for the first 9 questions when the participants are completing the ABTs in a room where temperature is set $17^{\circ} \mathrm{C}$ i.e. level 1 . Following the first nine questions, the average amount of time the participants take to answer each question 
remains approximately constant. This indicates that the participants require at least 9 questions to warm up for or get used to the SCWT.

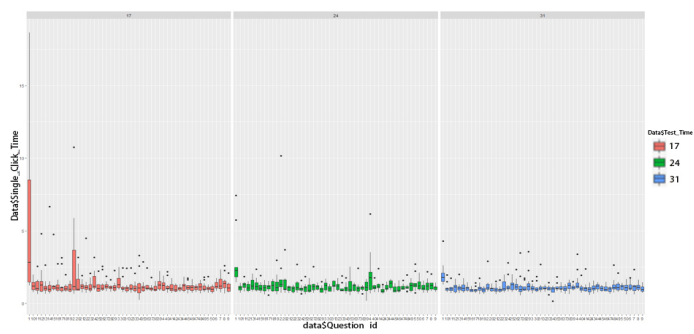

Fig. 15. Single click times for different question ID under three level of temperature

\subsubsection{The influence from gender.}

Fig. 16, Fig. 17 and Fig. 18 show the average amount of time the participants take to answer each question, splitting by genders i.e. male (1) and female (0), when they are completing the SCWT in a room with the temperature setting equal to $17^{\circ} \mathrm{C}, 24^{\circ} \mathrm{C}$ and $31{ }^{\circ} \mathrm{C}$ respectively.

From Fig. 16, Fig. 17 and Fig. 18, it can be noted that the average amount of time the female participants take to answer each question remains approximate constant under each level of temperature, and the average amount of time the male participants take to answer a question varies significantly in some questions when compared to the female's performance. When investigating the errors made in the SCWT, it is also interesting to note that errors are made by only female participants. This can be inferred that male participants are better at suppressing cognitive interference than females.

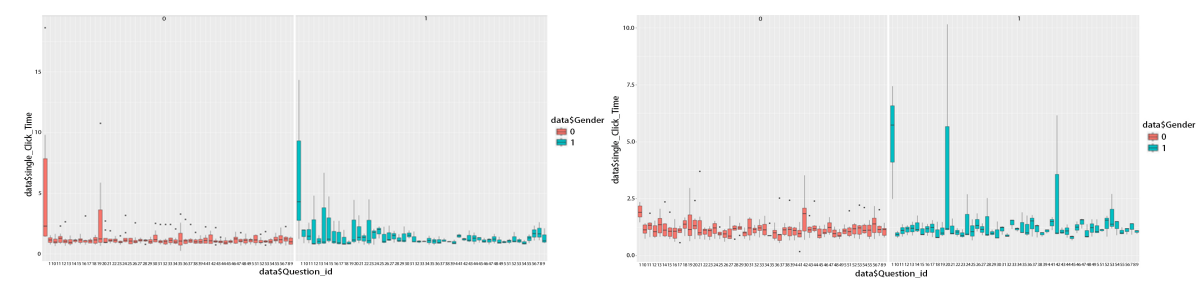

Fig. 16. The boxplot of single click time for different questions for female and male separately under the temperature of 17. Fig. 17. Under the temperature of 24.

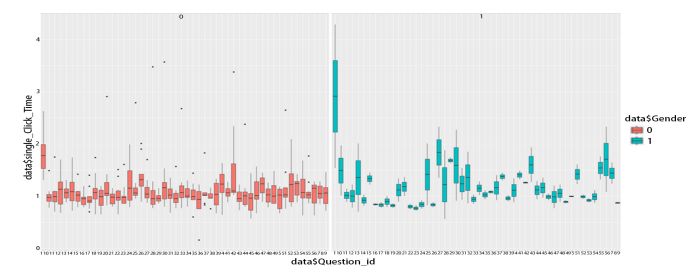

Fig. 18. The boxplot of single click time for different questions for female and male separately under the temperature of 31 . 


\subsubsection{Processing Speed of the Dot cancellation Task (DCT).}

Fig. 19 and Fig. 20 shows boxplot of processing speed for all participants when completing DCT under three different temperature settings. As shown in Fig. 19, most of the processing speed for all participants are between 0.5 and 0.9 number of 4 dots groups processed per second and the differences under the three temperature settings are barely noticeable. However, as shown in Fig. 20, significant differences exist as the temperature changes for some participants, and the tendency cannot be predicted.
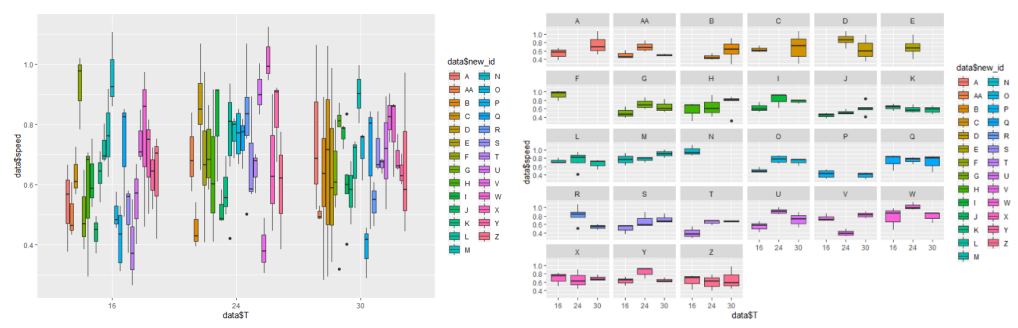

Fig. 19. The boxplot of data processing speed for each participant when they are tested under three different temperature Fig. 20. the boxplot of data processing speed for each participant when they are tested under three different temperature

\section{CONCLUSION}

The relationship between indoor temperature, noise and human attention level is investigated. Higher noise levels have negative impacts on the attention-level for the case of industrial noise, however, an opposite impact is true for the case of man-made and atmosphere noise types. The impact of different temperatures on the attention-level of the female participants are lower than the male participants. However, it is interesting to note that the ability to suppress cognitive interference are lower in females as indicated by the errors made during the SCWT.

According to DSM-5, restricted and repetitive patterns of behavior, interests or activities is a part of the condition. This manifests in four varieties including sensory aspects of the environment. Further investigations are required to evidence the feasibility of using off-the-shelf devices to develop a sensory management recommendation system for people with ASD. However, the preliminary assumption here is that the impact from temperature and noise will be even more noticeable than in the case of neurotypical people.

\section{$6 \quad$ Limitations}

There are 40 participants in total, including males and female with age range between 18 and 40. None of them were formally diagnosed with ASD. Some of the participants participated in both temperature and noise experiments, while some of the participants 
participated in either temperature or noise experiments. Participants were asked to familiarize themselves with the ABTs prior to their experiment. Participants of MTT were allowed to choose between a computer mouse and trackpad.

\section{References}

1. Cashin, A.; Barker, P. The triad of impairment in autism revisited. J. Child Adolesc. Psychiatr. Nurs. 2009, 22, 189-193.

2. Introduction to Sensorial. 2006. Montessori Primary Guide. Retrieve July 13, 2018 from http://www.info montessori.com/sensorial/introduction.htm

3. Developmental Disabilities Monitoring Network Surveillance Year 2010 Principal Investigators; Centers for Disease Control and Prevention (CDC) (2014) Prevalence of autism spectrum disorder among children aged 8 years - autism and developmental disabilities monitoring network, 11 sites, United States, 2010. MMWR Surveill Summ 63(2):1-21.

4. Roberta Costa Caminha, Carolina Lampreia. 2012. Findings on sensory deficits in autism: implications for understanding the disorder. Psychology \& Neuroscience. 5, 2: 231 - 237.

5. Ingram D, Takahashi N, Miles J. Defining autism subgroups: a taxometric solution. J Autism Dev Disord. 2008; 38:950-960. doi: 10.1007/s10803-007-0469-y.

6. Walker D, Thompson A, Zwaigenbaum L, Goldberg J, Bryson S, Mahoney W. Specifying PDD-NOS: a comparison of PDD-NOS, asperger syndrome, and autism. J Am Acad Child Adolesc Psychiatry. 2004;43:172-180. doi: 10.1097/00004583-200402000-00012.

7. Macintosh K, Dissanayake C. Annotation: the similarities and differences between autistic disorder and asperger's disorder: a review of the empirical evidence. J Child Psychol Psychiatry. 2004;45:421-434. doi: 10.1111/j.1469-7610.2004.00234.x.

8. Frazier T, Youngstrom E, Speer L, Embacher R, Law P, Constantino J. Validation of proposed DSM-5 criteria for autism spectrum disorder. J Am Acad Child Adolesc Psychiatry. 2012;51:28-40. doi: 10.1016/j.jaac.2011.09.021.

9. Huerta M, Bishop S, Duncan A, Hus V, Lord. Application of DSM-5 criteria for autism spectrum disorder to three samples of children with dsm-iv diagnoses of pervasive developmental disorders. Am J Psychiatry. 2012;169:1056-1064.

10. Grzadzinski R, Huerta M, Lord C. DSM-5 and autism spectrum disorders (ASDs): an opportunity for identifying ASD subtypes. Mol Autism. 2013;4(1):12. Published 2013 May 15.

11. G. Gillingham, Autism: Handle with Care: Understanding and Managing Behaviour of Children and Adults with Autism. Arlington: TX. Future Education Inc, 1995.

12. El Kaliouby, R. Affective computing and autism. Ann. N. Y. Acad. Sci. 2006, 1093, $228-$ 248.

13. Aresti-Bartolome, N., \& Garcia-Zapirain, B. (2014). Technologies as Support Tools for Persons with Autistic Spectrum Disorder: A Sys. Rev. Int. J. of Env. Res. \& Public Health, 11: 7767-7802.

14. Simmons, D. R., Robertson, A. E., McKay, L. S., Toal, E., McAleer, P. and F.E. Pollick: Vision in autism spectrum disorders. In: Vision Research, Vol. 49 (22), 2705-2739 (2009)

15. Gowen, E. and A. Hamilton: Motor abilities in autism: a review using a computational context. In: J. Autism Dev. Disord., vol. 43 (2), 323-344 (2013)

16. Otto-Meyer, S., Krizman, J., White-Schwoch, T. et al. Exp Brain Res (2018) 236: 733. https://doi-org.ezproxy.nottingham.edu.cn/10.1007/s00221-017-5164-4

17. Sound Meter Source LLC, (2018), available at: https://soundmetersource.com/index.html

18. Murphy, E. \& King, E. A. (2015), Testing the accuracy of smartphones and sound level meter applications for measuring environmental noise, Applied Acoustics, Elsevier. 
19. Murphy E, King E A. Testing the accuracy of smartphones and sound level meter applications for measuring environmental noise[J]. Applied Acoustics, 2016, 106:16-22.

20. App Store (2019), NIOSH Sound Level Meter, EA LAB.

21. Warren,S.F.;Gilkerson,J.;Richards,J.A.;Oller,D.K.;Xu,D.;Yapanel,U.;Gray,S.Whatautomatedvocal analysis reveals about the vocal production and language learning environment of young children with autism. J. Autism Dev. Disord. 2010, 40, 555-569.

22. Critchley, H.D. Electrodermal responses: What happens in the brain. Neuroscientist 2002, $8,132-142$.

23. Chen, W. and Cesar, P. (2015), Physiological Measurement on Students' Engagement in a Distributed Learning Environment.

24. Ruiz-Robledillo, Nicolás, Moya-Albiol L . Lower Electrodermal Activity to Acute Stress in Caregivers of People with Autism Spectrum Disorder: An Adaptive Habituation to Stress[J]. J. Autism Dev. Disord., 2015, 45(2):576-588.

25. Fenning R M, Baker J K, Baucom B R, et al. Electrodermal Variability and Symptom Severity in Children with Autism Spectrum Disorder[J]. J. Autism Dev. Disord., 2017, 47(4):111.

26. McCarthy, C.; Pradhan, N.; Redpath, C.; Adler, A. Validation of the Empatica E4 Wristband. In Proceedings of the 2016 IEEE EMBS International Student Conference (ISC), Ottawa, ON, Canada, 29-31 May 2016; 1-4.

27. Yekta,S.C;Niaz,C.;Deniz,E.;Cem,E.; Continuous Stress Detection Using Wearable Sensors in Real Life: Algorithmic Programming Contest Case Study, Sensors 2019, 19(8), 1849.

28. Taj-Eldin M , Ryan C , O' Flynn, Brendan, et al. A Review of Wearable Solutions for Physiological and Emotional Monitoring for Use by People with Autism Spectrum Disorder and Their Caregivers[J]. Sensors, 2018, 18(12).

29. Anderson, J.R. (2004), Cognitive Psychology and Its Implications (6th ed.), Worth Publishers, p. 519.

30. Hayden, S. (2014), Can this brain-sensing headband give you serenity, CNN, available at: http://edition.cnn.com/2014/08/18/tech/can-this-brain-sensing-headband/

31. Chawarska, K.; Macari, S.; Shic, F. Decreased spontaneous attention to social scenes in 6month-old infants later diagnosed with autism spectrum disorders. Biol. Psychiatry 2013, 74, 195-203.

32. Stroop J.R. "Studies of interference in serial verbal reactions". In: Experimental Psychology (1935).

33. Crawford J.R., Parker D.M., and McKinlay W.W. A Handbook of Neuropsychological Assessment.PsychologyPress, 1992.

34. Buscher G. et al. "Eye tracking analysis of preferred reading regions on the screen". In: In CHI '10 Extended Abstracts on Human Factors in Computing Systems (2010).

35. Budiu R (2018), Between-Subjects vs. Within-Subjects Study Design, available at: https://www.nngroup.com/articles/between-within-subjects/

36. Gao L (2005), Latin Squares in Experimental Design, available at: http://compneurosci. com/wiki/images/9/98/Latin_square_Method.pdf.

37. The Latin Square Design. url: https://newonlinecourses.science.psu.edu/ stat $503 /$ node $/ 21 /$. 